BMJ Open

Diabetes

Research

\& Care

\title{
Longitudinal associations between family conflict, parent engagement, and metabolic control in children with recent-onset type 1 diabetes
}

\author{
Hannah Case (D) , ${ }^{1}$ David D Williams, ${ }^{2}$ Shideh Majidi, ${ }^{3}$ Diana Ferro, ${ }^{2}$ \\ Mark Allen Clements, ${ }^{4}$ Susana R Patton ${ }^{5}$
}

\begin{abstract}
To cite: Case H, Williams DD, Majidi S, et al. Longitudinal associations between family conflict, parent engagement, and metabolic control in children with recentonset type 1 diabetes. BMJ Open Diab Res Care 2021;9:e002461. doi:10.1136/ bmjdrc-2021-002461
\end{abstract}

Received 29 June 2021 Accepted 26 September 2021

Check for updates

\section{(c) Author(s) (or their} employer(s)) 2021. Re-use permitted under CC BY-NC. No commercial re-use. See rights and permissions. Published by BMJ.

${ }^{1}$ Mayo Clinic Alix School of Medicine, Mayo Clinic Hospital Jacksonville, Jacksonville,

Florida, USA

${ }^{2}$ Division of Health Services and Health Outcomes Research, Children's Mercy Hospitals and Clinics, Kansas City, Missouri, USA

${ }^{3}$ Barbara Davis Center for Diabetes and the University of Colorado Anschutz School of Medicine, University of Colorado - Anschutz Medical Campus, Aurora, Colorado, USA ${ }^{4}$ Pediatrics, Endocrinology, Children's Mercy Hospitals and Clinics, Kansas City, Missouri, USA

${ }^{5}$ Center for Healthcare Delivery Science, Nemours Children's Health System, Jacksonville, Florida, USA

Correspondence to Dr Susana R Patton; Susana.Patton@nemours.org

\section{ABSTRACT}

Introduction We prospectively investigated the associations between diabetes-related family conflict, parent engagement in child type 1 diabetes (T1D) care, and child glycated hemoglobin ( $\mathrm{HbA1C}$ ) in 127 families of school-age children who we recruited within the first year of their T1D diagnosis.

Research design and methods Parents completed the Diabetes Family Conflict Scale-Revised (DFCS-R) to assess for diabetes-related family conflict and the Diabetes SelfManagement Questionnaire-Brief (DSMQ-Brief) to assess parent engagement in child T1D care at the initial study visit (T1) and at 12 (T2) and 27 (T3) months later. We also collected child $\mathrm{HbA1C}$ at these time points. Our analyses included Pearson correlations and repeated measures linear mixed models controlling for child age, sex, and T1D duration at T1.

Results Parents' DFCS-R scores negatively correlated with DSMQ-Brief scores $(r=-0.13, p<0.05)$ and positively correlated with children's HbA1c $(r=0.26, p<0.001)$. In our linear mixed models, parents' DSMQ-Brief scores were unchanged at $\mathrm{T} 2(\beta=-0.71,95 \% \mathrm{Cl}-1.59$ to 0.16$)$ and higher at $\mathrm{T} 3(\beta=8.01,95 \% \mathrm{Cl} 6.89$ to 9.13$)$ compared with $\mathrm{T} 1$, and there was an association between increasing DFCS-R and decreasing DSMQ-Brief scores $(\beta=-0.14$, $95 \% \mathrm{Cl}-0.21$ to -0.06$)$. Child $\mathrm{HbA1c}$ values were significantly higher at T2 $(\beta=0.66,95 \% \mathrm{Cl} 0.38$ to 0.94$)$ and $\mathrm{T} 3(\beta=0.95,95 \% \mathrm{Cl} 0.63$ to 1.27$)$ compared with $\mathrm{T} 1$, and there was an association between increasing DFCS-R scores and increasing child $\mathrm{HbA} 1 \mathrm{C}(\beta=0.04,95 \% \mathrm{Cl} 0.02$ to 0.06 ).

Conclusions Increasing diabetes-specific family conflict early in T1D may associate with decreasing parent engagement in child T1D care and increasing child HbA1c, suggesting a need to assess and intervene on diabetesspecific family conflict.

Trial registration number NCT03698708.

\section{INTRODUCTION}

Youth with type 1 diabetes (T1D) experience dramatic reductions in glycated hemoglobin (HbA1c) levels after initiation of insulin therapy, ${ }^{1}$ but glycemic levels tend to increase in many youth over the next 12-24 months. ${ }^{2}$ Prior studies have addressed associations

\section{Significance of this study}

What is already known about this subject?

- Prior studies have demonstrated associations between diabetes-specific family conflict and suboptimal glycemic levels and self-care engagement in families of adolescents and families of youth who are not in the recent-onset period of type 1 diabetes (T1D).

What are the new findings?

- In this 27-month prospective longitudinal study, parents of young school-age children with recentonset T1D reported clinically noteworthy levels of diabetes-specific family conflict that increased with time and associated with decreasing parent engagement in child T1D care and increasing child glycemic levels over time.

- Because diabetes-specific family conflict is potentially treatable, our findings highlight a new target for future clinical intervention for these families.

How might these results change the focus of research or clinical practice?

- Diabetes care teams should consider screening for diabetes-specific conflict among families of young school-age children with recent-onset T1D as it appears to associate with suboptimal changes in parent engagement in child T1D care and child glycemic levels.

between increasing glycemic levels and both loss of residual beta cell function (also known as the 'honeymoon period') and the onset of puberty in youth. ${ }^{34}$ Yet few studies have examined the relationship between parent/child factors and longitudinal child glycemic levels in the first 24 months after families establish diabetes care, a period that might reflect the experiences and outcomes of children with recent-onset T1D.

The recent-onset period of T1D is a unique time for parents and children. ${ }^{5}$ First, this period requires rapid knowledge and skill 
acquisition for T1D management in parents and children, including glucose monitoring, insulin administration, and carbohydrate counting. Second, it is a period of T1D when children are often vulnerable to wide fluctuations in their blood glucose levels because of residual beta cell function. ${ }^{1}$ According to international guidelines, ${ }^{6}$ it is recommended for parents of children with recentonset T1D to oversee and even complete much of the daily T1D management on behalf of their child, which could underscore the importance of examining parent/ child factors as they relate to children's glycemia. For families of youth with T1D who are beyond 24 months postdiagnosis, studies suggest that diabetes-specific family conflict may be an important parent/child factor to include when examining child glycemic levels. ${ }^{7-13}$ Diabetes-specific family conflict is a construct that reflects how much a parent and child with T1D argue about T1D management. ${ }^{14}$ Related, it may be important to include a measure of either youth or parent engagement with T1D care to assess how often parents or youth are participating in T1D management. ${ }^{12} 15$ There are many crosssectional studies conducted in adolescents with T1D that report associations between higher diabetes-specific family conflict and suboptimal youth engagement in T1D care and HbAlc. ${ }^{9-12} 15$ There is also a study conducted in families of adolescents with T1D which found a longitudinal association between diabetes-specific family conflict and youth HbA1c that was partially mediated by youth engagement in T1D care. ${ }^{16}$

As a possible explanation for why diabetes-specific family conflict could relate to T1D engagement and youth HbA1c, it may be that parent-child conflict around diabetes self-care tasks and shared responsibility of those tasks impedes parents' beneficial involvement and collaboration with their youth's T1D care, leading to suboptimal HbA1c levels. ${ }^{815}$ In this way, diabetes-specific family conflict, which is increasingly recognized as a potentially modifiable factor in diabetes management, could also be an important clinical target for intervention. ${ }^{781015}$ For families of younger children with T1D and families of children with recent-onset T1D, we know far less about how diabetes-specific family conflict may relate to suboptimal T1D engagement or child HbAlc and this could represent a critical gap in knowledge related to the clinical management of these families.

Therefore, this study expands on previous literature by investigating the association between family conflict, parent engagement in child T1D care, and HbAlc in a sample of children recently diagnosed with T1D. Compared with prior studies, the patient cohort examined here is younger (mean age of 7 years), closer to their T1D diagnosis (within 12 months), and the study design includes a longer follow-up period (up to 27 months) to assure data collection beyond the recentonset period. We specifically selected a measure of parent engagement in child T1D care versus child engagement because we anticipated parents would play a significant role in their child's T1D care. Precisely, we hypothesized that parents would report diabetes-specific family conflict despite their child's young age and minimal time since T1D onset and that increasing family conflict over time would associate with decreasing parent engagement in child T1D care and increasing child HbAlc levels.

\section{METHODS}

\section{Participants and procedures}

We recruited parents and children aged 5-9 years old and within 12 months of their T1D diagnosis at two pediatric diabetes clinics in the USA to participate in a prospective longitudinal study examining psychosocial factors that may contribute to youth glycemic levels during the recent-onset period of T1D (TACKLE: Treatment Adherence and Control in Kids: Longitudinal Evaluation).

Eligible families had a child within 12 months of his or her T1D diagnosis, who was between 5 and 9 years old, used intensive insulin therapy (insulin pump or multiple daily injections), and spoke English. Families excluded from participation were families of children diagnosed with a developmental delay, severe psychiatric disorder, or comorbid chronic condition, families of children with type 2 or monogenic diabetes, and children taking medications that may impact glycemic levels.

Parents provided informed consent and permission for their child to participate. Children $\geq 7$ years old provided assent; this included any child who turned 7 years old while participating in the longitudinal study. For each parent and child dyad, we collected child HbAlc levels and parent survey responses at the initial study visit (T1) and at 12 and 27 months later (T2 and T3, respectively). We targeted parents who self-identified as assuming a primary role in the daily management of their child's T1D and had the same parent complete surveys at each time point. While the recent-onset period may conclude at 24 months, we include data at 27 months to assure that we are crossing the threshold beyond the recent-onset period with one data collection point in the "established diabetes' period. We compensated parents for participation at each study visit using a clin card or gift card. Children received a toy valued at US $\$ 10$ for completing an HbA1c kit at each time point.

\section{Measures}

\section{Demographics}

Parents reported all demographic information at the initial study visit (T1). This included child age, child sex, race/ethnicity, duration of $\mathrm{T} 1 \mathrm{D}$, caregiver relation to child, caregiver age, caregiver marital status, and family income.

\section{Hemoglobin A1c}

We collected children's HbA1c by a finger-stick blood sample and capillary tube collection at all study visits (T1T3). We analyzed all study samples at a central laboratory using automated high-performance liquid chromatography (reference range 4.0\%-6.0\% (20-42 $\mathrm{mmol} / \mathrm{mol})$; Tosoh Corporation, San Francisco, California). ${ }^{17}$ In the 
rare event of a missing or diluted laboratory sample, we used children's most current point-of-care HbA1c values. Results suggested $>0.90$ reliability between children's available point-of-care and laboratory-based HbA1c.

\section{Diabetes Family Conflict}

Parents used the Diabetes Family Conflict Scale-Revised (DFCS-R $)^{14}$ to report on their perceptions of diabetesspecific family conflict. Parents completed this survey at all study visits (T1-T3). Items of the DFCS-R identify 19 T1D management tasks, such as remembering to give shots or bolus for insulin and recognizing symptoms of a low blood glucose and telling other people about diabetes. According to the standardized instructions, parents responded to items using a Likert scale based on how often they argue with their child with T1D about each behavior (from $1=$ never argue to $3=$ always argue).${ }^{14}$ We scored parents' DFCS-R surveys based on total scores (range: 19-57), with higher scores reflecting greater levels of conflict. The DFCS-R is valid for use in parents of children with T1D and appropriate for administration in a research or clinical setting. ${ }^{14}$

\section{Diabetes Self-Management}

The Diabetes Self-Management Questionnaire-Brief (DSMQ-Brief $)^{18}$ is a nine-item measure of engagement in T1D care over the past month. It includes items specific to insulin use (How often have you administered insulin more than 30 minutes late or forgotten to administer it at all?), glucose monitoring (How often did you check blood glucose within 30 minutes before a meal?), diet (Do you usually count carbs, measure, or weigh food, or use exchanges to figure out how much to give?), and exercise (When your child got a different amount of exercise than usual, how often did you make changes in the meal plan or insulin amount?). ${ }^{18}$ Parents responded to each item to characterize their level of engagement in their child's T1D care, with higher scores corresponding to higher levels of parent engagement. The DSMQ-Brief scores can range from 6 to 35. Parents completed this survey at all study visits (T1-T3). The DSMQ-Brief is valid for use in parents of children with T1D and in research and clinical settings. ${ }^{18}$

\section{Statistical analysis}

We performed descriptive and analytic statistical analyses using the Stata/SE V.15.1. We adopted an alpha of $\mathrm{p}<0.05$ to denote statistical significance and report $95 \%$ CI.

Our descriptive statistics included frequencies, percentages, means, and SD of our demographic and outcome data. We calculated Pearson correlations for the DSMQ-Brief, DFCS-R, and child HbAlc. To test our primary hypothesis, we used repeated measures linear mixed models (LMMs) to evaluate associations over time (T1-T3) between parents' DSMQ-Brief scores and child HbA1c with parents' DFCS-R total scores. For these models, we controlled for child initial study visit age, child sex, and duration of T1D, and we used participant as the fixed effect.

\section{RESULTS}

\section{Sample characteristics}

The analyzed sample included 127 parent-child dyads. At the initial study visit (T1), children had a mean time since diagnosis of $4.7 \pm 3.3$ months and a mean age of $7.8 \pm 1.3$ years. There were $65(51 \%)$ girls and $62(49 \%)$ boys in the sample. Parents in the study were predominantly mothers $(87 \%)$ and had a mean age of $36.6 \pm 6.4$ years at T1. The parent-child dyads self-reported their race and ethnicity. The sample was $84 \%$ white, $10 \%$ black/ African American, and $6 \%$ preferring not to report race. Of the parents, $11 \%$ also indicated that they identified as Hispanic/Latinx. Parents self-reported family income. Approximately $50 \%$ of the sample reported a household income at or below the 2019 US median income of $\$ 68$ $703 .^{19}$

\section{Measures and outcomes descriptives}

At the initial study visit (T1), parents' mean DFCS-R total score was $23.18 \pm 6.15$, parents' DMSQ-Brief score was $27.77 \pm 3.88$, and child HbA1c was $7.61 \% \pm 1.37 \%$. At 12 months (T2), parents' mean DFCS-R total score was 24.78 \pm 6.68 , parents' DMSQ-Brief score was 26.78 \pm 4.40 , and child HbAlc was $8.26 \% \pm 1.23 \%$. At 27 months (T3), parents' mean DFCS-R total score was $25.35 \pm 6.42$, parents' DMSQ-Brief score was 35.49 \pm 5.31 , and child HbA1c was $8.57 \% \pm 1.40 \%$.

\section{Bivariate correlations}

Across time points, parents' DFCS-R total scores negatively correlated with their DSMQ-Brief scores $(\mathrm{r}=-0.13$, $\mathrm{p}<0.05$ ) and positively correlated with children's HbA1c levels $(\mathrm{r}=0.26, \mathrm{p}<0.001)$. Figures 1 and 2 show parents' DSMQ-Brief scores and child HbA1c values, respectively, and DFCS-R total scores at T1-T3.

\section{Linear mixed models}

In a series of LMMs, we explored the associations of parents' DFCS-R total scores with parent-reported DSMQBrief scores and children's HbAlc levels, while controlling for time, child age and duration of T1D at T1, and child sex. In the first model, there was a time effect for families' DSMQ-Brief scores. Compared with T1, parent-reported DSMQ-Brief scores were unchanged at T2 ( $\beta=-0.71,95 \%$ CI -1.59 to 0.16$)$ and significantly higher at T3 $(\beta=8.01$, $95 \%$ CI 6.89 to 9.13 ). However, consistent with the study hypothesis, we observed a significant effect of parentreported DFCS-R total scores, such that for each unit increase in conflict there was 0.14 decrease in parentreported DSMQ-Brief scores $(\beta=-0.14,95 \%$ CI -0.21 to -0.06$)$. In the second model, there was a significant time effect for children's HbAlc levels. Compared with $\mathrm{T} 1$, child HbA1c values were significantly higher at T2 ( $\beta=0.66,95 \%$ CI 0.38 to 0.94$)$ and T3 $(\beta=0.95,95 \%$ CI 0.63 to 1.27 ). Also consistent with the study hypothesis, we observed a statistically significant association between increasing DFCS-R total scores and increasing child HbA1c levels, such that for each unit increase in conflict 

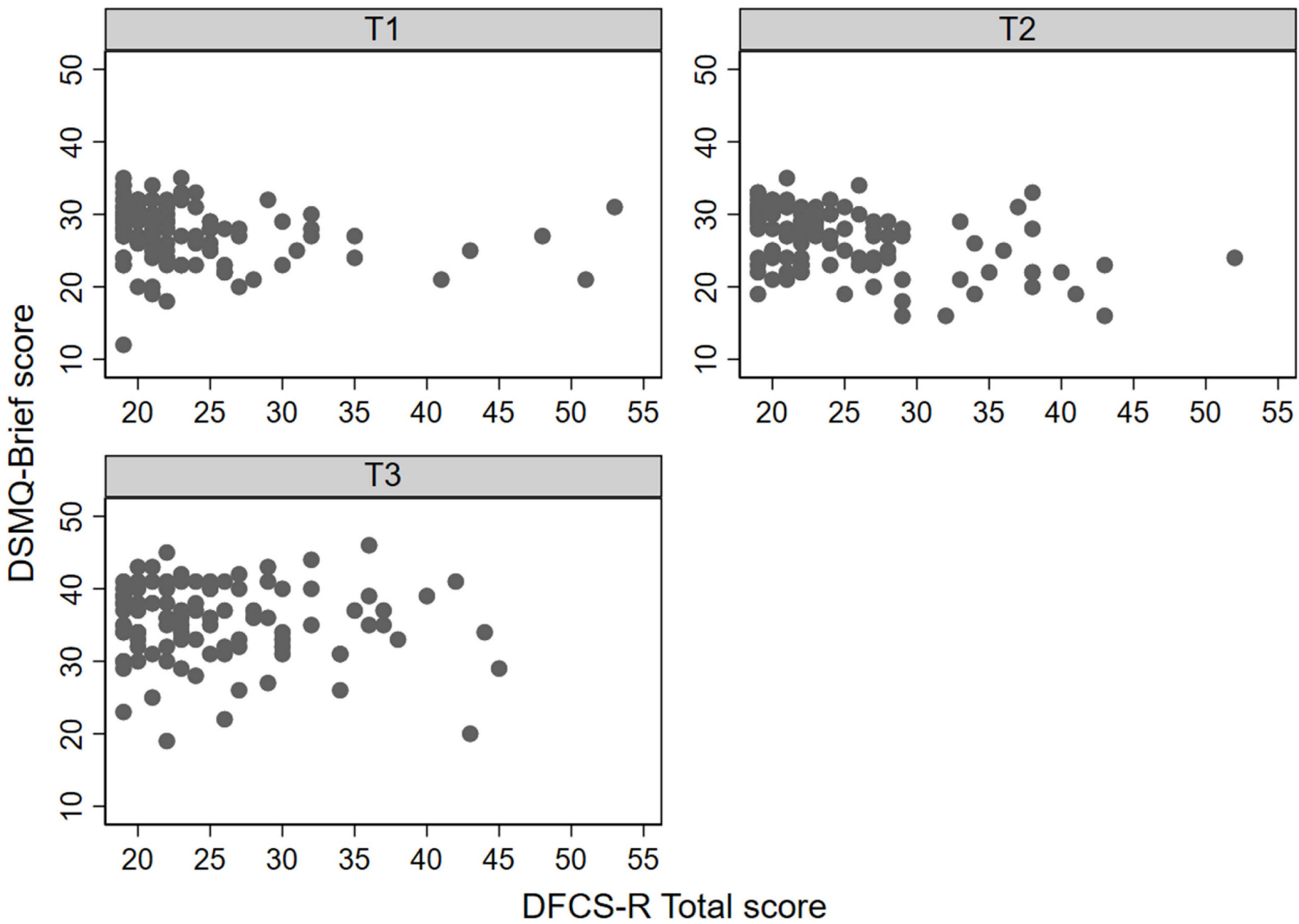

Figure 1 Parents' DSMQ-Brief scores and DFCS-R total scores at initial study visit (T1), at 12 months (T2), and at 27 months (T3). DFCS-R, Diabetes Family Conflict Scale-Revised; DSMQ-Brief, Diabetes Self-Management Questionnaire-Brief.

there was $0.04 \%$ increase in child HbA1c $(\beta=0.04,95 \%$ CI 0.02 to 0.06 ).

\section{DISCUSSION}

In a sample of families of young school-age children with recent-onset $\mathrm{T} 1 \mathrm{D}$, we found that parents reported the presence of diabetes-specific family conflict and that their perceptions of conflict increased over the study period. Specifically, at T1, parents of young school-age children with recent-onset T1D reported levels of diabetesspecific family conflict that were comparable with levels reported by parents of adolescents with T1D..$^{11} 121416$ This is clinically important because any level of family conflict can undermine parent-child collaboration to perform diabetes care tasks as well as impact their perceptions of quality of life. ${ }^{111214}$ Related, the nearly two-point increase in parents' mean diabetes-specific family conflict scores between T1 and T3 is also clinically important as it highlights the possibility that families who report greater diabetes-specific family conflict close to diabetes onset will report increasing conflict over time as families transition from recent-onset to established T1D care periods.

Focusing on our primary hypothesis, in a series of repeated measures LMMs that controlled for time, child age, time since diagnosis, and child sex, our results suggested longitudinal associations between parentreported diabetes-specific family conflict such that increasing conflict related to decreasing parent engagement in child T1D care. We also saw a longitudinal association between increasing diabetes-specific family conflict and increasing child HbAlc. Thus, the results of our LMMs highlight how diabetes-specific family conflict could negatively affect families. If parents and children are arguing more during the recent-onset period, it is possible this conflict could make it harder for them to establish effective parent-child collaboration for diabetes care, thereby leading to decreasing perceptions of parent engagement in T1D care and increasing child HbAlc. As noted earlier, other studies have found similar associations between family conflict, youth engagement in T1D care, and HbAlc in adolescents, ${ }^{8-121516}$ but to our knowledge this is the first time research has provided evidence showing these longitudinal associations in children younger than adolescents and the first time documenting these associations up to 2.5 years beyond diabetes onset for children.

We also found significant time effects for parent engagement in child T1D care and child HbA1c. Notably, 


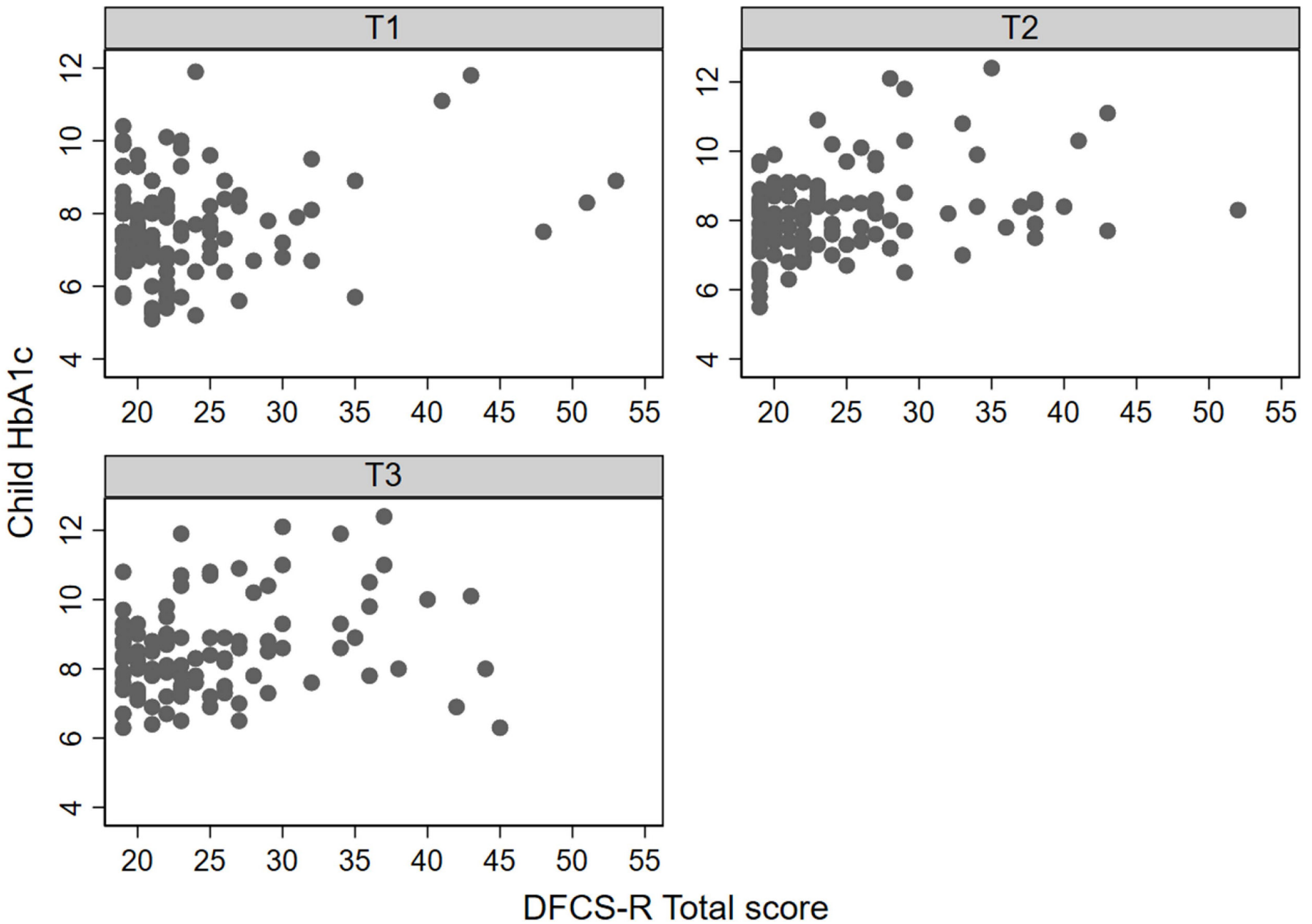

Figure 2 Children's HbA1c and parents' DFCS-R total scores at initial study visit (T1), at 12 months (T2), and at 27 months (T3). DFCS-R, Diabetes Family Conflict Scale-Revised; HbA1c, glycated hemoglobin.

the time effect for parent engagement in child T1D care suggested that parents reported increasing DSMQ-Brief scores between $\mathrm{T} 1$ and T3. This result may provide additional evidence to support the notion that families will do better with diabetes self-care when they have adequate knowledge and resources to start off with more optimal levels of parent engagement in child T1D care. The time effect for child HbAlc suggested that children with higher HbA1c levels at $\mathrm{T} 1$ also had increasing HbAlc levels at T2 and T3, suggesting that even early in T1D some children may experience a trajectory of increasing HbA1c levels that might translate into greater vulnerability for acute (such as diabetes ketoacidosis) and longterm sequela of suboptimal glucose levels (including nephropathy, neuropathy, and retinopathy). ${ }^{20}$

Also, with regard to the temporal effects for child HbAlc, it may be important to consider the potential role of the honeymoon period. ${ }^{1}$ While previous studies have presupposed the end of the honeymoon period to be one explanation for why children may experience an increase in their HbAlc during the recent-onset period, ${ }^{1-3}$ increasing diabetes-specific family conflict could offer an alternative hypothesis and a modifiable target. It is possible that parents and children could perceive greater stress and conflict if the loss of residual endogenous insulin secretion results in greater effort to appropriately care for T1D (ie, the end of the honeymoon period contributes to increasing conflict). Similarly, it is possible the presence of conflict could hamper effective communication about diabetes care, leading to a decrease in parent engagement in child T1D care and rising child HbAlc levels (ie, increasing conflict reduces parent engagement in child T1D care, contributing to rising HbA1c). Considering these results, one clinical implication may be to initiate screening for diabetes-specific family conflict during the recent-onset period. The DFCS- ${ }^{14}$ is a short survey, validated for use in parents and youth, which may be scalable for in clinic use. Moreover, for families who screen positive for conflict, there are successful interventions that reduce family conflict by improving family communication, collaboration, and empathy. ${ }^{21-24}$ Thus, it is possible early detection and treatment for diabetes-specific conflict could help families of young school-age children to establish a beneficial collaboration for daily parent engagement in child T1D care and to achieve optimal HbA1c levels closer to diagnosis. 
Although the insights contributed by this study are clinically meaningful, we recognize study limitations. These include the lack of racial and ethnic diversity of the sample, the potential over-reliance on mothers' perspectives, the use of a parent-report measure of parent engagement in child T1D care versus objective assessment via frequency of self-monitoring blood glucose or insulin Bolus scores, ${ }^{25}$ and potential confounding due to differences in when each child exited the honeymoon period. In the future, it will be important to examine these associations in a cohort of families of school-age children with recent-onset T1D from diverse racial and ethnic backgrounds to increase generalizability of the results. Indeed, increasing evidence draws attention to the impact of long-standing health, social, and economic inequities in the USA on child glycemic levels, making it essential to pursue additional studies to better understand how diabetes-specific family conflict is associated with child HbA1c. ${ }^{26}$ Our study procedures enabled us to recruit any parent who self-identified as providing primary care for their child's T1D management, although we primarily had mothers participating. In the future, studies should attempt to solicit perceptions from fathers or other caregivers. ${ }^{727}$ Another focus of future research should be to confirm our study findings using objective measures of parent engagement in child T1D care, such as the days of continuous glucose monitor use and children's insulin Bolus score. ${ }^{25}$ Similarly, future studies should include the methodology to assess for biomarkers (eg, C peptide) that may signal residual beta cell function to personalize how long young school-age children with T1D are experiencing the honeymoon effect and how exiting this period specifically relates to parents' perceptions of diabetesspecific family conflict, parent engagement in child T1D care, and children's HbA1c levels. ${ }^{62}$ Strengths of this study include its prospective, longitudinal design, with repeated assessments up to 27 months poststudy enrollment, its relatively large cohort of children with recent-onset T1D, its use of a central laboratory to analyze the study-specific HbAlc samples, and its use of validated parent-report surveys.

In conclusion, this study highlights the importance of assessing diabetes-specific family conflict, especially in the context of optimizing glycemic levels and encouraging optimal parent engagement in child T1D care among families of young school-age children with recent-onset T1D. We believe that it is sustainable to screen for diabetes-specific family conflict using validated surveys. Moreover, because conflict is potentially modifiable, ${ }^{21-24}$ it may be a promising novel target for interventions that aim to improve glycemic levels for children experiencing increasing HbAlc in the recent-onset period. Future studies should be aimed at assessing implementation of screening for diabetesspecific family conflict in the recent-onset period and the efficacy of interventions to help parents and children establish a beneficial collaboration for T1D care tasks and a trajectory of optimal child HbA1c.

Contributors $\mathrm{HC}$ and SRP wrote the manuscript. SRP designed the research study and secured funding. DDW analyzed the data. MAC and SM assisted with study execution and provided feedback on the manuscript. DF provided feedback on the manuscript.

Funding This research was supported in part by grant R01-DK100779 (PI: SRP) from the National Institutes of Health/National Institute of Diabetes and Digestive and Kidney Diseases.

Competing interests MAC is the chief medical officer for Glooko and has consulted with Medtronic Diabetes and Eli Lilly on topics unrelated to the submitted work. All other authors have nothing to disclose.

Patient consent for publication Not required.

Ethics approval The study was approved by the Children's Mercy Hospital Institutional Review Board (IRB\#14030130) and the Colorado Multiple Institutional Review Board (IRB\#14-1356).

Provenance and peer review Not commissioned; externally peer reviewed.

Data availability statement Data are available upon reasonable request. De-identified data that support the findings of this study are available from the corresponding author (SRP) upon reasonable request.

Open access This is an open access article distributed in accordance with the Creative Commons Attribution Non Commercial (CC BY-NC 4.0) license, which permits others to distribute, remix, adapt, build upon this work non-commercially, and license their derivative works on different terms, provided the original work is properly cited, appropriate credit is given, any changes made indicated, and the use is non-commercial. See: http://creativecommons.org/licenses/by-nc/4.0/.

ORCID iD

Hannah Case http://orcid.org/0000-0003-4097-9500

\section{REFERENCES}

1 Abdul-Rasoul M, Habib H, Al-Khouly M. 'The honeymoon phase' in children with type 1 diabetes mellitus: frequency, duration, and influential factors. Pediatr Diabetes 2006;7:101-7.

2 Cengiz E, Cheng P, Ruedy KJ, et al. Clinical outcomes in youth beyond the first year of type 1 diabetes: results of the pediatric diabetes Consortium (PDC) type 1 diabetes new onset (neon) study. Pediatr Diabetes 2017;18:566-73.

3 Prahalad P, Yang J, Scheinker D, et al. Hemoglobin A1c trajectory in pediatric patients with newly diagnosed type 1 diabetes. Diabetes Technol Ther 2019;21:456-61.

4 Schwandt A, Kuss O, Dunstheimer D, et al. Three-Variate longitudinal patterns of metabolic control, body mass index, and insulin dose during puberty in a type 1 diabetes cohort: a groupbased Multitrajectory analysis. J Pediatr 2020;218:64-71.

5 Marker AM, Monzon AD, Goggin K, et al. Parent perspectives on educational and psychosocial intervention for recent-onset type 1 diabetes in their school-age child: a qualitative study. Diabetes Spectr 2021;34:166-74.

6 Couper JJ, Haller MJ, Greenbaum CJ, et al. ISPAD clinical practice consensus guidelines 2018: stages of type 1 diabetes in children and adolescents. Pediatr Diabetes 2018;19 Suppl 27:20-7.

7 Fox DA, Bone JN, Keidar S, et al. Family conflict in type 1 diabetes: who is at risk? Pediatr Diabetes 2020;21:1575-82.

8 Vaid E, Lansing AH, Stanger C. Problems with self-regulation, family conflict, and glycemic control in adolescents experiencing challenges with managing type 1 diabetes. J Pediatr Psychol 2018;43:525-33.

9 Savin KL, Hamburger ER, Monzon AD, et al. Diabetes-specific family conflict: informant discrepancies and the impact of parental factors. J Fam Psychol 2018;32:157-63.

10 Anderson BJ, Vangsness L, Connell A, et al. Family conflict, adherence, and glycaemic control in youth with short duration type 1 diabetes. Diabet Med 2002;19:635-42.

11 Williams LB, Laffel LMB, Hood KK. Diabetes-specific family conflict and psychological distress in paediatric type 1 diabetes. Diabet Med 2009;26:908-14.

12 Noser AE, Huffhines L, Clements MA, et al. Diabetes conflict outstrips the positive impact of self-efficacy on youth adherence and glycemic control in type 1 diabetes. Pediatr Diabetes 2017;18:614-8. 
13 Campbell MS, Wang J, Cheng Y, et al. Diabetes-specific family conflict and responsibility among emerging adults with type 1 diabetes. J Fam Psychol 2019;33:788-96.

14 Hood KK, Butler DA, Anderson BJ, et al. Updated and revised diabetes family conflict scale. Diabetes Care 2007;30:1764-9.

15 Miller VA, Drotar D. Discrepancies between mother and adolescent perceptions of diabetes-related decision-making autonomy and thei relationship to diabetes-related conflict and adherence to treatment. J Pediatr Psychol 2003;28:265-74.

16 Hilliard ME, Guilfoyle SM, Dolan LM, et al. Prediction of adolescents glycemic control 1 year after diabetes-specific family conflict: the mediating role of blood glucose monitoring adherence. Arch Pediatr Adolesc Med 2011;165:624-9.

17 Khuu HM, Robinson CA, Goolsby K, et al. Evaluation of a fully automated high-performance liquid chromatography assay for hemoglobin A1c. Arch Pathol Lab Med 1999;123:763-7.

18 Markowitz JT, Laffel LMB, Volkening LK, et al. Validation of an abbreviated adherence measure for young people with type 1 diabetes. Diabet Med 2011;28:1113-7.

19 Semega A, Kollar M, Shrider EA. Income and poverty in the United States: 2019, 2019. United States census bureau. Available: Income and Poverty in the United States: 2019 (census.gov) [Accessed 08/2021].

20 Patton SR, Feldman K, Majidi S, et al. Identifying $\mathrm{HbA1c}$ trajectories and modifiable risk factors of trajectories in 5- to 9-year-olds with recent-onset type 1 diabetes from the United States. Diabet Med 2021;38:e14637.
21 Hood KK, Hilliard M, Piatt G, et al. Effective strategies for encouraging behavior change in people with diabetes. Diabetes Manag 2015;5:499-510.

22 McBroom LA, Enriquez M. Review of family-centered interventions to enhance the health outcomes of children with type 1 diabetes. Diabetes Educ 2009;35:428-38.

23 Wysocki T, Harris MA, Buckloh LM, et al. Randomized trial of behavioral family systems therapy for diabetes: maintenance of effects on diabetes outcomes in adolescents. Diabetes Care 2007;30:555-60.

24 Wysocki T, Harris MA, Buckloh LM, et al. Effects of behavioral family systems therapy for diabetes on adolescents' family relationships, treatment adherence, and metabolic control. J Pediatr Psychol 2006;31:928-38.

25 Patton SR, Clements MA, Fridlington A, et al. Frequency of mealtime insulin bolus as a proxy measure of adherence for children and youths with type 1 diabetes mellitus. Diabetes Technol Ther 2013;15:124-8.

26 Willi SM, Miller KM, DiMeglio LA, et al. Racial-ethnic disparities in management and outcomes among children with type 1 diabetes. Pediatrics 2015;135:424-34.

27 Hilliard ME, Rohan JM, Carle AC, et al. Fathers' involvement in preadolescents' diabetes adherence and glycemic control. J Pediatr Psychol 2011;36:911-22.

28 Fonolleda M, Murillo M, Vázquez F, et al. Remission phase in paediatric type 1 diabetes: new understanding and emerging biomarkers. Horm Res Paediatr 2017;88:307-15. 